\title{
Phase I single and multiple dose study to evaluate the safety, tolerability, and pharmacokinetics of BMS-927711 in healthy subjects
}

\author{
G Tong, I Savant, N Jariwala, D Burt, N Zheng, A Buzescu, R Bertz, S Keswani, R Marcus ${ }^{*}$ \\ From The European Headache and Migraine Trust International Congress \\ London, UK. 20-23 September 2012
}

\section{Introduction}

Calcitonin gene-related peptide (CGRP) may play a causal role in migraine. Blocking the CGRP receptor may be an effective approach to migraine relief while avoiding the vasoconstrictive effects associated with triptans. BMS-927711 is a potent, selective, CGRP receptor antagonist. The

\section{Objective}

of this Phase I study was to assess the safety, tolerability, and pharmacokinetics of single (SAD) and multiple ascending (MAD) oral doses of BMS-927711 in healthy subjects.

\section{Methods}

For SAD, 8 healthy subjects received a single dose $(25,75,150,300,600,900$, or $1500 \mathrm{mg})$ of BMS-927711 or placebo. For MAD, 8 healthy subjects received a daily dose $(75,150,300,450$, and $600 \mathrm{mg})$ or 300 twice daily of BMS-927711 or placebo for 14 days.

\section{Results}

BMS-927711 was well tolerated at single doses up to 1500 $\mathrm{mg}$ and at multiple doses up to $600 \mathrm{mg}$ for 14 days. There were no serious adverse events (AEs). The maximum tolerated dose was not reached. The most common AEs among BMS-927711 groups during SAD were nausea $(\mathrm{n}=7$ BMS; $\mathrm{n}=0$ placebo, and dizziness $(\mathrm{n}=5$ BMS; $\mathrm{n}=0$ placebo), and were constipation ( $\mathrm{n}=8$ BMS; $\mathrm{n}=3$ placebo) and headache ( $\mathrm{n}=8$ BMS; $\mathrm{n}=2$ placebo) during MAD. Most AEs were mild in nature. 2 subjects discontinued the
MAD due to skin rash, and 1 discontinued due to elevated creatinine. BMS-927711 antagonizes CGRP-induced increases in marmoset facial blood flow (a surrogate marker for intracranial artery dilation) with $75 \%$ inhibition at $\sim 700 \mathrm{nM}$, a surrogate for efficacious exposure. Following single doses, the plasma exposures exhibited biphasic disposition with a terminal T1/2 of $\sim 10-12$ hrs. At 2 hours (SAD), $\geq 700 \mathrm{nM}$ exposure was achieved in most subjects at doses $\geq 75 \mathrm{mg}$. Food delayed Tmax (from 1 to 4 hrs post dose), and famotidine coadministration reduced bioavailability (Cmax $\sim 26 \%$, AUC $\sim 42 \%$ ).

\section{Conclusions}

BMS-927711 appeared to be safe and well tolerated over a range of doses in this healthy population. After single oral doses $\geq 75 \mathrm{mg}$, clinical exposures are above the efficacious margin of $700 \mathrm{nM}$ at 2 hrs post dose, supporting further clinical development in acute migraine.

Published: 21 February 2013

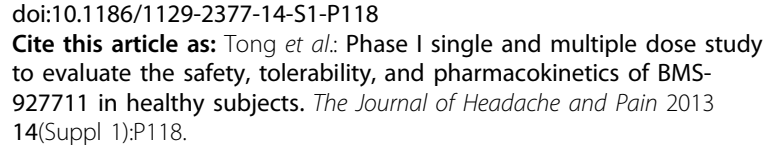

